\title{
ANALYTIC CONTINUATION OF EISENSTEIN SERIES
}

\author{
BY
}

\author{
JOSEPH LEWITTES $\left({ }^{1}\right)$
}

\begin{abstract}
The classical Eisenstein series are essentially of the form $\sum_{m, n}^{\prime}\left(\left(m+r_{1}\right) z+n+r_{2}\right)^{-s}, m, n$ ranging over integer values, $\operatorname{Im} z>0, r_{1}, r_{2}$ rational and $s$ an integer $>2$. In this paper we show that if $s$ is taken to be complex the series, with $r_{1}, r_{2}$ any real numbers, defines an analytic function of $(z, s)$ for $\operatorname{Im} z>0, \operatorname{Re} s>2$. Furthermore this function has an analytic continuation over the entire $s$ plane, exhibted explicitly by a convergent Fourier expansion. A formula for the transformation of the function when $z$ is subjected to a modular transformation is obtained and the special case of $s$ an integer is studied in detail.
\end{abstract}

Introduction. In this paper we are concerned with the function $G\left(z, s, r_{1}, r_{2}\right)$ defined as the sum of the series $\Sigma_{m, n}^{\prime} 1 /\left(\left(m+r_{1}\right) z+n+r_{2}\right)^{s}$, for $z$ having positive imaginary part, $s$ an arbitrary complex number and $r_{1}, r_{2}$ arbitrary real numbers. $m, n$ range over all integer values. In $\$ I$ we define which branch of the 'multi-valued' complex power should be taken, and show that although the series converges only for $\operatorname{Re} s>2, G$ has an analytic continuation to all values of $s$. This is done by exhibiting a Fourier expansion for $G$ convergent for all $s$. For $r_{1}, r_{2}$ rational numbers and $s$ an integer $\geq 3$ one sees that $G$ coincides essentially with the classical Eisenstein series; this then is the reason for the title. A few more details on this will be found in $\$$ III. In $\$ I I$ we determine how $G$ transforms when $z$ is subjected to a transformation $z \rightarrow(a z+b) /(c z+d)$ belonging to the modular group.

To put this paper in proper perspective we remark that its significance is not just in the formulas in $\$$ II but also in that considering $G$ as a function of the variable $s$, several classical functions are obtained for special values of $s$ and $r_{1}, r_{2}$, each of whose functional equations has always been derived separately while here they all appear as specializations of one general formula. Thus, besides the above mentioned Eisenstein series for $s=k \geq 3, r_{1}, r_{2}$, rational, we also obtain in $\$$ III Hecke's generalized Eisenstein series for $s=2$, while the case $s=r_{1}=r_{2}=0$ gives us the transformation formula for $\log \eta, \eta$ being Dedekind's $\eta$ function.

Received by the editors September 30, 1971.

AMS 1970 subject classifications. Primary 10D05; Secondary 30A14, 30A 16, 30A 58.

Key words and phrases. Analytic continuation, Eisenstein series, modular group, zeta function.

(1) Partially supported by NSF GP 9466. 
In a previous paper [2] we considered the special case $r_{1}=r_{2}=0$ and only the modular transformation $z \rightarrow-1 / z$. However, the present paper is independent of [2], the latter's results being included here as particular instances of the general case.

I. We use the standard notations $Z, R, C$, for the integers, real numbers, complex numbers, respectively. $z=x+i y, w=u+i v, s=\sigma+i t$ are complex variables, $\mathcal{H}$ the upper half plane $\{y>0\}, \mathcal{H}_{-}$the lower half plane $\{y<0\}$ and $\mathcal{K}_{\sigma_{0}}$ the right half plane $\left\{\sigma>\sigma_{0}\right\}$. The letters $k, m, n, M, N$ always take on integer values and a sign such as $\Sigma_{m}$ indicates summation as $m$ ranges over all integer values. $\chi$ is the characteristic function of the integers, $\chi(z)=1$ if $z \epsilon$ $Z$ and $\chi(z)=0$ if $z \notin Z$. For real $x,[x]$ is the greatest integer $\leq x$ and $\langle x\rangle=$ $x-[x]$, the fractional part of $x$. If $z \in C, z \neq 0, \operatorname{Arg} z$ is the set of real numbers $\theta$ for which $z=|z| e^{i \theta}$; we say then $\theta$ is a value of $\operatorname{Arg} z$. We recall that $\operatorname{Arg} z$ is not empty and if $\theta$ is a value of $\operatorname{Arg} z$, then $\phi$ is also if, and only if, $\phi=\theta+$ $2 \pi k, k$ an integer. There is a unique $\theta \in \operatorname{Arg} z$ satisfying $-\pi \leq \theta<\pi$, which we call $\arg z$, the argument of $z$. Logarithm and power are defined, for $z \neq 0$ and all $s$, by $\log z=\log |z|+i \arg z$ and $z^{s}=e^{s \log z}$. Note that $z^{-s}=1 / z^{s}$, and $\left|z^{s}\right|=$ $e^{-t \arg z}|z|^{\sigma} \leq e^{\pi|t|}|z|^{\sigma}$. The following lemma collects some facts about the argument function that will be useful later.

Lemma 1. (a) Let $\theta_{j}=\arg z_{j}$ for $j=1,2$. Then

$$
\arg z_{1} z_{2}= \begin{cases}\theta_{1}+\theta_{2}, & \text { if }-\pi \leq \theta_{1}+\theta_{2}<\pi, \\ \theta_{1}+\theta_{2}+2 \pi, & \text { if } \theta_{1}+\theta_{2}<-\pi, \\ \theta_{1}+\theta_{2}-2 \pi, & \text { if } \theta_{1}+\theta_{2} \geq \pi .\end{cases}
$$

(b) Let $A, B, C, D$ be real, $A, B$ not both zero and $C$ positive.

Then for $z \in \mathcal{H}$,

$$
\arg \left(\frac{A z+B}{C z+D}\right)=\arg (A z+B)-\arg (C z+D)+2 \pi k,
$$

where $k$ is an integer independent of $z \in \mathcal{H}$ depending on $A, B, C, D$ as follows:

$$
\begin{aligned}
& k=1, \quad \text { if } A \leq 0, A D-B C>0, \\
& k=0, \quad \text { otherwise. }
\end{aligned}
$$

Proof. (a) $\theta_{1}+\theta_{2} \in \operatorname{Arg} z_{1} z_{2}$ so that $\arg z_{1} z_{2}=\theta_{1}+\theta_{2}+2 \pi k, k$ the unique integer satisfying $-\pi \leq \theta_{1}+\theta_{2}+2 \pi k<\pi$. Since $-\pi \leq \theta_{1}, \theta_{2}<\pi,-2 \pi \leq \theta_{1}+$ $\theta_{2}<2 \pi$, and $k$ can be only 0,1 or -1 . (1) now follows directly by considering the possible cases.

(b) Clearly (2) holds with $k$ an integer depending upon $z$. But for $z \in \mathcal{H}$ each of $(A z+B) /(C z+D), A z+B, C z+D$, takes values in only one of $\mathcal{H}, \mathcal{H}_{-}$, the positive real numbers, or the negative real numbers, and the argument function re- 
stricted to any one of these sets is continuous. Thus the integer valued function $k=k(z)$ defined by (2) is a continuous function of $z \in \mathcal{H}$, hence constant and independent of $z$ as asserted. In (2) now, set $z=(i-D) / C \in \mathcal{H}$, since $C>0, E=$ $-A D+B C, w=E / C+i A / C$, and upon solving for $2 \pi k,(2)$ becomes, $2 \pi k=$ $\arg (-i) w-\arg w+\pi / 2$. Computing $\arg (-i) w$ by (1) gives (3).

Special cases of (1) used frequently and without comment are: If $z \neq 0, u$ real $>0$, then $\arg u z=\arg z,(u z)^{s}=u^{s} z^{s} ;$ if $z \in \mathcal{H}$ or $z$ real $>0$ and $u$ real $>0$, then $\arg (-u z)=\arg z-\pi$ and $(-u z)^{s}=e^{-\pi i s} u^{s} z^{s}$.

We now introduce the series which is the basic concern of this paper. Let $\left(r_{1}, r_{2}\right) \in R^{2}$ and consider

$$
\sum_{m, n}^{\prime} \frac{1}{\left(\left(m+r_{1}\right) z+n+r_{2}\right)^{s}},
$$

the accent on $\Sigma$ indicating that we omit any value of $(m, n) \in Z^{2}$ which gives an undefined term. This convention will be used often, it generally being clear from the context which values are to be omitted. In (4), for example, $(m, n)=$ $\left(-r_{1},-r_{2}\right)$ is omitted. Of course, this is a restriction only in case $\left(r_{1}, r_{2}\right) \in Z^{2}$. It is known, and not difficult to prove, that the series (4) converges uniformly absolutely on compact subsets of $(z, s) \in \mathcal{H} \times \mathcal{K}_{2}$. Because of the absolute convergence there is no need to specify the order of the terms in the series, all arrangements add up to the same sum. Futhermore each individual term of the series is an analytic function of $(z, s) \in \mathcal{H} \times C$ so that the sum defines an analytic function $G$ on $\mathcal{H} \times \mathcal{K}_{2}$ which we denote as $G\left(z, s, r_{1}, r_{2}\right)$. It is clear that $G$ depends only on the coset of $\left(r_{1}, r_{2}\right) \bmod Z^{2}$; changing $r_{1}, r_{2}$ by integers does not change $G$.

Theorem 1. $G$ bas an everywhere finite analytic continuation to the entire $s$ plane given by the formula, valid for $(z, s) \in \mathcal{H}_{\times} \mathcal{K}_{2}$ :

$$
\begin{aligned}
& \sum_{m, n}^{\prime} \frac{1}{\left(\left(m+r_{1}\right) z+n+r_{2}\right)^{s}} \\
& =\chi^{\left(r_{1}\right)} \sum_{n}^{\prime} \frac{1}{\left(n+r_{2}\right)^{s}}+\frac{(-2 \pi i)^{s}}{\Gamma(s)} \sum_{m>-r_{1} \cdot k=1} \sum^{\infty} k^{s-1} \exp \left(2 \pi i k r_{2}\right) \exp \left(2 \pi i k\left(m+r_{1}\right) z\right) \\
& +e^{\pi i s} \frac{(-2 \pi i)^{s}}{\Gamma(s)} \sum_{m>r_{1}} \sum_{k=1}^{\infty} k^{s-1} \exp \left(-2 \pi i k r_{2}\right) \exp \left(2 \pi i k\left(m-r_{1}\right) z\right) .
\end{aligned}
$$

The two double series on the right side of (5) converge uniformly absolutely on compact subsets of $\mathcal{H} \times C$ defining analytic functions of $(z, s) \in \mathcal{H} \times C$ and the first series on the right side of (5) also bas an everywhere finite analytic continuation over the entire $s$ plane. 
Proof. Let

$$
P(z, s)=\sum_{n}^{\prime} \frac{1}{(z+n)^{s}}
$$

in which $z \in C$ is arbitrary and the accent indicates that the value $n=-z$ is omitted-again, this is an actual restriction only in case $z$ is an integer. It is easy to see that the series converges absolutely as long as $\sigma>1$ and for fixed $z$ the convergence is uniform on compact subsets of $K_{1}$. Thus for each fixed $z, P(z, s)$ is an analytic function on $K_{1}$. However, for fixed $s$, not an integer, $P(z, s)$ is not a continuous function of $z$; for example,

$$
\lim _{y \rightarrow 0+} \sum_{n}\left(\frac{1}{2}+i y+n\right)^{-s} \neq \sum_{n}\left(\frac{1}{2}+n\right)^{-s},
$$

and this of course is due to the discontinuity of the argument function on the negative real axis. Thus we introduce the following three functions: $F=P$ restricted to $\mathcal{H}_{\times} \mathcal{K}_{1}, F_{-}=P$ restricted to $\mathcal{H}_{-} \times K_{1}$, and $F_{0}=P$ restricted to $R \times K_{1}$. In (6) each individual term is analytic on $\mathcal{H}_{\times} C$ and $\mathcal{H}_{-} \times C$ and the absolute convergence is uniform on compact subsets of $\mathcal{H} \times \mathcal{K}_{1}$ so that $F$ and $F_{\text {_ }}$ are analytic functions of $(z, s)$ on their respective domains.

The function $F_{0}(x, s)$ is closely related to the Hurwitz or generalized $\zeta$ function defined usually only for $0<x \leq 1, \sigma>1$ by $\zeta(s, x)=\sum_{n=0}^{\infty}(x+n)^{-s}$. We find it convenient to consider it defined for all $x$ by $\zeta(s, x)=\Sigma_{n>-x}(x+n)^{-s}$. Then $\zeta(s, x)=\zeta(s,\langle x\rangle)=\zeta(s, x+k)$ for all integers $k$ and $\zeta(s, k)=\zeta(s)$, the Riemann $\zeta$ function, for all integers $k$. To be consistent with our previous notation we should write $\zeta(x, s)$, but we shall adhere to tradition and write $\zeta(s, x)$. The reason is that one wishes to give prominence to $s$ in which $\zeta$ is analytic rather than $x$ which enters as a parameter. From its definition $\zeta(s, x)$ is analytic in $s \in K_{1}$ and it is known that it has a continuation over the entire $s$ plane having only a simple pole at $s=1$ with residue 1 and everywhere else finite. This and other facts about the $\zeta$ function used in this paper can be found in [3, Chapter XIIIl.

Now we have

$$
F_{0}(x, s)=\sum_{n>-x}(x+n)^{-s}+\sum_{n<-x}(x+n)^{-s}=\zeta(s, x)+e^{\pi i s} \zeta(s,-x) .
$$

This shows that $F_{0}$ can be continued over the $s$ plane, and is everywhere finite, for at $s=1, \zeta(s, x)=1 /(s-1)+\cdots, e^{\pi i s}=-e^{\pi_{i(s-1)}}=-1+\cdots$, so $\zeta(s, x)+$ $e^{\pi i s} \zeta(s,-x)=(1 /(s-1)+\cdots)+(-1+\cdots)(1 /(s-1)+\cdots)$, where $\cdots$ represent higher order terms in $(s-1)$ and so the $1 /(s-1)$ terms cancel each other out.

Concerning $F$ and $F_{-}$, if $z \in \mathcal{H}_{-}$, then $-z \in \mathcal{H}$ and $(z+n)^{-s}=$ 
$e^{\pi i s}(-z-n)^{-s}$ so that $F_{-}(z, s)=\Sigma_{n}(z+n)^{-s}=e^{\pi i s} \Sigma_{n}(-z+n)^{-s}=$ $e^{\pi i s} F(-z, s)$ for as $n$ ranges over $Z$ so does $-n$. Thus

$$
F_{-}(z, s)=e^{\pi i s} F(-z, s)
$$

and the properties of $F_{-}$can be deduced from those of $F$.

Since $F(z+1, s)=F(z, s), F$ has a Fourier expansion in $z$ :

$$
F(z, s)=\sum_{k} a_{k}(s) e^{2 \pi i k z}
$$

We recall that this is 'obtained by setting $w=e^{2 \pi i z}$ and noting that because of the periodicity of $F$ in $z$, the function $f$ defined by $f(w, s)=F(z, s)$ is a single valued analytic function of $w$ in the punctured disk $0<|w|<1$, thus having a Laurent expansion $\Sigma_{k} a_{k} w^{k}$ uniformly convergent on compact subsets of the punctured disk. This gives (9). Furthermore, $a_{k}(s)=(1 / 2 \pi i) \int_{|w|=r}\left(f(w, s) / w^{k+1}\right) d w$, $0<r<1$, which after carrying out the substitution $w=e^{2 \pi i z}$ and replacing $F$ by its defining series gives

$$
a_{k}(s)=\int_{I} e^{-2 \pi i k z} \sum_{n}(z+n)^{-s} d z
$$

where $I$ is any interval in $\mathcal{H}$ of length 1 parallel to the real axis, say, for definiteness, $I=\{0 \leq x \leq 1, y=1\}$. Now, as the series is uniformly convergent on $I$, interchange $\int$ and $\Sigma$, then in the $n$th integral make the change of variable $z=$ $z^{\prime}-n$, which shifts the interval of integration to $\{n \leq x \leq n+1, y=1\}$, collect the integrals and finally one has

$$
a_{k}(s)=\int_{L} z^{-s} e^{-2 \pi i k z} d z
$$

where $L$ is the line $\{-\infty<x<\infty, y=1\}$. This integral is perhaps not completely elementary and is of basic importance to our development so we carry out the evaluation. The fact that $k$ is an integer plays no role.

Lemma 2. Let $L$ be the line $\{-\infty<x<\infty, y=1\}$, ib a real number, $\sigma>1$, $a_{b}(s)=\int_{L} z^{-s} e^{-2 \pi i b z} d z$. Then

$$
\begin{aligned}
a_{b}(s) & =0 & & \text { if } b \leq 0, \\
& =(-2 \pi i)^{s} b^{s-1} / \Gamma(s) & & \text { if } b>0 .
\end{aligned}
$$

Proof. For $R>0$ let $C_{R}$ be the circle $|z|=R$ and for $R>1, L_{R}$, the segment of $L$ cut off by $C_{R}$, which is just the interval from $-R^{\prime}+i$ to $R^{\prime}+i$ where $R^{\prime}=\left(R^{2}-1\right)^{1 / 2} \cdot \int_{L}=\lim _{R \rightarrow \infty} \int_{L_{R}}$ and since $z^{-s} e^{-2 \pi i b z}$ is analytic in $\mathcal{H}, \int_{L_{R}}=\int_{S_{R}}, S_{R}$ being the arc of $C_{R}$ lying above $L$. If $b \leq 0$, then on $S_{R}$, $\left|z^{-s} e^{-2 \pi i b z}\right| \leq e^{\pi|t|} R^{-\sigma},\left|\int_{L_{R}}\right|=\left|\int_{S_{R}}\right| \leq e^{\pi|t|} R^{-\sigma} \int_{S_{R}}|d z|<2 \pi e^{\pi|t|} R^{1-\sigma} \rightarrow 0$ as $R \rightarrow \infty$, yielding the first part of (12). 
For $b>0, e^{-2 \pi i b z}$ is unbounded on $S_{R}$ as $R \rightarrow \infty$ so we turn to the arc of $C_{R}$ lying below $L$. But now we must take into account the discontinuity of $z^{-s}$ on the negative real axis before we can use the residue theorem. It is convenient to shift this discontinuity to the negative imaginary axis-or $-y$ axis, for short-so for the purpose at hand only, define

$$
\begin{aligned}
\left\{z^{-s}\right\} & =z^{-s} & & \text { if }-\pi / 2<\arg z<\pi, \\
& =e^{-2 \pi i s} z^{-s} & & \text { if }-\pi \leq \arg z<-\pi / 2 ;
\end{aligned}
$$

$\left\{z^{-s}\right\}$ is the power of $z$ obtained by using that $\theta \in \operatorname{Arg} z$ satisfying $-\pi / 2<\theta<$ $3 \pi / 2$. Now $\left\{z^{-s}\right\}$ is analytic in the $z$ plane slit along the $-y$ axis and $a_{b}(s)=$ $\int_{L}\left\{z^{-s}\right\} e^{-2 \pi i b z} d z$. Let $E_{-}, E_{+}$denote the 'left' and 'right' 'edges', respectively, of the $-y$ axis and define $\left\{z^{-s}\right\}$ as $e^{-2 \pi i s} z^{-s}$ on $E_{-}$and as $z^{-s}$ on $E_{+}$, these values being determined by considerations of continuity upon approach from the left or right. The method we are using is standard in the calculus of residues, easily justified, so we do not go into all the subsidiary details. Let $\gamma_{R}$ be the curve starting at $-R^{\prime}+i$, going a long $C_{R}$ to $-i R$, up $E_{-}$to $-i r, 0<r<1$, clockwise around $C_{r}$, back down $E_{+}$to $-i R$ and then along $C_{R}$ to $R^{\prime}+i$. Then $\int_{L_{R}} z^{-s} e^{-2 \pi i b z} d z=\int_{\gamma_{R}}\left\{z^{-s}\right\} e^{-2 \pi i b z} d z$ and we can estimate this latter integral. Let $a$ be the angle subtended by the arc of $C_{R}$ from $R$ to $R^{\prime}+i ; 0<a<\pi / 2$, $\sin \alpha=1 / R$. The contribution of the arcs of $C_{R}$ to $\int_{\gamma_{R}}$ is

hence

$$
J_{R}=\int_{\pi-a}^{3 \pi / 2}+\int_{-\pi / 2}^{a} R^{-s} e^{-i s \theta} \exp \left(-2 \pi i b R e^{i \theta}\right) R e^{i \theta} i d \theta
$$

$$
\left|J_{R}\right| \leq e^{2 \pi|t|} R^{1-\sigma}\left(\int_{\pi-a}^{3 \pi / 2}+\int_{-\pi / 2}^{a}\right) e^{2 \pi b R \sin \theta} d \theta .
$$

But $0<\int_{\pi}^{3 \pi / 2}+\int_{-\pi / 2}^{0} e^{2 \pi b R \sin \theta} d \theta<\pi$, since $b>0$ so we will have $J_{R} \rightarrow 0$ as $R \rightarrow \infty$ if we show $\int_{\pi-a}^{\pi} \int_{0}^{a} e^{2 \pi b R} \operatorname{sir} \theta d \theta=2 \int_{0}^{a} e^{2 \pi b R} \sin \theta d \theta$ is a bounded function of $R$. Indeed, though, for $0 \leq \theta \leq \alpha, 0 \leq R \sin \theta \leq R \sin \alpha=1$ and $\int_{0}^{a} e^{2 \pi b R \sin \theta} d \theta \leq \int_{0}^{a} e^{2 \pi b} d \theta=e^{2 \pi b} \alpha \rightarrow 0$ as $R \rightarrow \infty$. Having disposed of the contribution of $C_{R}$ as $R \rightarrow \infty$ we have now attained $\int_{L}=\int_{\gamma}$ where $\gamma$ is $E_{-}$ starting at $-i \infty$ up to $-i r$, clockwise around $C_{r}$, then back down $E_{+}$from - ir to $-i_{\infty}$. On $E_{-}, z=-i y, y>0,\left\{z^{-s}\right\}=e^{-3 \pi i s / 2} y^{-s}$ while on $E_{+}, z=-i y$, $y>0,\left\{z^{-s}\right\}=e^{\pi i s / 2} y^{-s}$ and a small calculation gives

$$
\int_{\gamma}=-i\left(e^{\pi i s / 2}-e^{-3 \pi i s / 2}\right) \int_{r}^{\infty} y^{-s} e^{-2 \pi b y} d y-\int_{|z|=r}\left\{z^{-s}\right\} e^{-2 \pi i b z} d z .
$$

Each of these integrals is easily seen to be convergent for all $s$, giving an analytic continuation of $\int_{\gamma}$ to all $s$ values. For $\sigma<1$, the obvious estimates show that $\int|z|=r \rightarrow 0$ as $r \rightarrow 0$, so that 


$$
\begin{aligned}
\int_{\gamma} & =e^{-\pi i s / 2} \frac{\left(e^{\pi i s}-e^{-\pi i s}\right)}{i} \int_{0}^{\infty} y^{-s} e^{-2 \pi b y} d y \\
& =(-i)^{s} 2 \sin \pi s(2 \pi b)^{s-1} \Gamma(1-s)=\frac{(-2 \pi i)^{s}}{\Gamma(s)} b^{s-1},
\end{aligned}
$$

where in the last two steps we used Euler's integral for $\Gamma(1-s)$ and the functional equation $\sin (\pi s) \Gamma(1-s)=\pi / \Gamma(s)$. Since $\int_{\gamma}$ and $(-2 \pi i)^{s} b^{s-1} / \Gamma(s)$ are both entire functions and agree for $\sigma<1$, they are equal for all $s$. This completes the proof of the lemma.

For brevity, let

$$
\Delta(s)=(-2 \pi i)^{s} / \Gamma(s)
$$

$\Delta$ is an entire function with simple zeros at $s=0$ or a negative integer. Taking account of (8), (9), and (12) we have

$$
\begin{gathered}
F(z, s)=\Delta(s) \sum_{k=1}^{\infty} k^{s-1} e^{2 \pi i k z}, \\
F_{-}(z, s)=e^{\pi i s} \Delta(s) \sum_{k=1}^{\infty} k^{s-1} e^{-2 \pi i k z} .
\end{gathered}
$$

These series converge uniformly absolutely on compact subsets of $\mathcal{H} \times C, \mathcal{H}_{-} \times$ $C$ respectively-in fact, the smaller $\sigma$ the more rapid the convergence-thus giving analytic continuations for $F, F$, over the whole $s$ plane. Returning now to $G$, defined as the sum of (4) for $(z, s) \in \mathcal{H} \times K_{2}$, we break up the sum as follows:

$$
\sum_{m, n}^{\prime}=\chi^{\left(r_{1}\right)} \sum_{m=-r_{1}} \sum_{n}^{\prime}+\sum_{m>-r_{1}} \sum_{n}+\sum_{m<-r_{1}} \sum_{n} \text {. }
$$

Now

$$
\sum_{m=-r_{1}} \sum_{n}^{\prime}=\sum^{\prime}\left(n+r_{2}\right)^{-s}=\zeta\left(s, r_{2}\right)+e^{\pi i s} \zeta\left(s,-r_{2}\right)
$$

as remarked in the start of the proof. The other double series, using the definitions of $F, F_{-}$and then (14), (15), give

$$
\begin{aligned}
\sum_{m>-r_{1}} \sum_{n}\left(\left(m+r_{1}\right) z+n+r_{2}\right)^{-s}=\sum_{m>-r_{1}} F\left(\left(m+r_{1}\right) z+r_{2}, s\right) \\
=\Delta(s) \sum_{m>-r_{1}} \sum_{k=1}^{\infty} k^{s-1} \exp \left(2 \pi i k r_{2}\right) \exp \left(2 \pi i k\left(m+r_{1}\right) z\right)
\end{aligned}
$$


and

$$
\begin{aligned}
\sum_{m<-r_{1}} \sum_{n}\left(\left(m+r_{1}\right) z+n+r_{2}\right)^{-s} & =\sum_{m<-r_{1}} F_{-}\left(\left(m+r_{1}\right) z+r_{2}, s\right) \\
& =e^{\pi i s} \Delta(s) \sum_{m<-r_{1}} \sum_{k=1}^{\infty} k^{s-1} \exp \left(-2 \pi i k r_{2}\right) \exp \left(-2 \pi i k\left(m+r_{1}\right) z\right) .
\end{aligned}
$$

In the last sum over $m<-r_{1}$ if we set $m=-m^{\prime}, m^{\prime}>r_{1}$ and then call $m^{\prime}$ again $m$, we have

$$
e^{\pi i s} \Delta(s) \sum_{m>r_{1}} \sum_{k=1}^{\infty} k^{s-1} \exp \left(-2 \pi i k r_{2}\right) \exp \left(2 \pi i k\left(m-r_{1}\right) z\right)
$$

Putting all this into (16) completes the proof of (5). Now for the statements about analytic continuation, those involving the $\zeta$ function have been dealt with already. We only have to show that the double series converge for all $s$. If we define the function $A$ by these double series:

$$
A\left(z, s, r_{1}, r_{2}\right)=\sum_{m>-r_{1}} \sum_{k=1}^{\infty} k^{s-1} \exp \left(2 \pi i k r_{2}\right) \exp \left(2 \pi i k\left(m+r_{1}\right) z\right)
$$

we see that (5) can be written

$$
\begin{aligned}
G\left(z, s, r_{1}, r_{2}\right)= & \chi\left(r_{1}\right)\left(\zeta\left(s, r_{2}\right)+e^{\pi i s} \zeta\left(s,-r_{2}\right)\right) \\
& +\Delta(s)\left(A\left(z, s, r_{1}, r_{2}\right)+e^{\pi i s} A\left(z, s,-r_{1},-r_{2}\right)\right) .
\end{aligned}
$$

Now if we take the absolute value of each term in (17) we obtain

$$
\sum_{m>-r_{1}} \sum_{k=1}^{\infty} k^{\sigma-1} \exp \left(-2 \pi k\left(m+r_{1}\right) y\right)
$$

If $r_{1} \in Z$ then $m=-\quad+q, q=1,2, \ldots$, and we have

$$
\begin{aligned}
\sum_{q=1}^{\infty} \sum_{k=1}^{\infty} k^{\sigma-1} e^{-2 \pi k q y} & =\sum_{n=1}^{\infty}\left(\sum_{k \mid n} k^{\sigma-1}\right) e^{-2 \pi n y} \\
& \leq \sum_{n=1}^{\infty} n^{\sigma} e^{-2 \pi n y} \\
& \leq \sum_{n=1}^{\infty} n^{\sigma}{ }^{\sigma} \exp \left(-2 \pi n y_{0}\right)<\infty,
\end{aligned}
$$

for $\sigma \leq \sigma_{0}, y \geq y_{0}>0$. If $r_{1} \notin Z$, then $m=-\left[r_{1}\right]+q, q=0,1,2, \cdots, m+r_{1}=$ $\left.q+\left\langle r_{1}\right\rangle,\left\langle r_{1}\right\rangle\right\rangle 0$, and the series of absolute values is 


$$
\begin{aligned}
\sum_{q=0}^{\infty} \sum_{k=1}^{\infty} k^{\sigma-1} \exp \left(-2 \pi k\left(q+\left\langle r_{1}\right\rangle\right) y\right) & \\
= & \sum_{k=1}^{\infty} k^{\sigma-1} \exp \left(-2 \pi k\left\langle r_{1}\right\rangle y\right)+\sum_{q=1}^{\infty} \sum_{k=1}^{\infty} k^{\sigma-1} \exp \left(-2 \pi k\left\langle r_{1}\right\rangle y\right) e^{-2 \pi k q y} \\
& <\sum_{k=1}^{\infty} k^{\sigma-1} \exp \left(-2 \pi k\left\langle r_{1}\right\rangle y\right)+\sum_{q=1}^{\infty} \sum_{k=1}^{\infty} k^{\sigma-1} e^{-2 \pi k q y}
\end{aligned}
$$

and again we see the convergence is uniform for $\sigma \leq \sigma_{0}, y \geq y_{0}>0$. Thus $A\left(z, s, r_{1}, r_{2}\right)$ is analytic for $(z, s) \in \mathcal{H} \times C$, and like $G$ depends only on the coset of $\left(r_{1}, r_{2}\right) \bmod Z^{2}$. The proof of the theorem is now finished.

Later on we shall be particularly interested in the case where $s$ is an integer. One immediate consequence of (18) is that if $s=\nu, \nu$ an integer $\leq 0$,

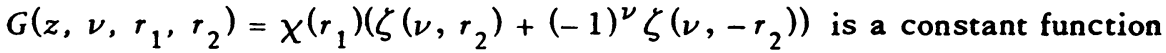
of $z$ (see (45) below for evaluation of this constant). For, $\Delta(\nu)=0$ and the $A$ 's are always finite. If $\left(r_{1}, r_{2}\right) \in Z^{2}$, then $G\left(z, s, r_{1}, r_{2}\right)=G(z, s, 0,0)=G(z, s)$, for short. Similarly we set $A(z, s)=A(z, s, 0,0)$. Then (17) gives

$$
A(z, s)=\sum_{n=1}^{\infty} \sigma_{s-1}(n) e^{2 \pi i n z}
$$

where $\sigma_{s-1}(n)=\Sigma_{k \mid n} k^{s-1}$. In this instance, also standard notation, the symbol $\sigma$ is not the real part of $s$ as elsewhere. Then (18) simplifies to

$$
G(z, s)=\left(1+e^{\pi i s}\right)\left(\zeta(s)+(-2 \pi i)^{s} A(\bar{z}, s) / \Gamma(s)\right) .
$$

From this we see that $G(z, 1)$ is also a constant function of $z$, its value being $\left.\left(1+e^{\pi i s}\right) \zeta(s)\right|_{s=1}=-\pi i$. Also for $\nu \leq 0, G(z, \nu)$ is by above $\left(1+(-1)^{\nu}\right) \zeta(\nu)$ which is 0 if $\nu<0$ and -1 if $\nu=0$. Indeed, $\zeta(0)=-1 / 2$ while if $\nu<0,1+$ $(-1)^{\nu}=0$ if $\nu$ is odd, and $\zeta(\nu)=0$ if $\nu$ is even.

In any event then, $G$ always gives a constant function of $z$ for $s$ a nonpositive integer, so that from a certain point of view $A$ is the more interesting function. In particular we note the intriguing fact that $A(z, 0)$ is closely related to a classical function, the Dedekind $\eta$ function. We recall that this is defined by

$$
\eta(z)=e^{\pi i z / 12} \prod_{m=1}^{\infty}\left(1-e^{2 \pi i m z}\right),
$$

for $z \in \mathcal{H}$. Then

$$
\begin{aligned}
\log \eta(z)-\pi i z / 12 & =\sum_{m=1}^{\infty} \log \left(1-e^{2 \pi i m z}\right)=-\sum_{m=1}^{\infty} \sum_{k=1}^{\infty} \frac{e^{2 \pi i m k z}}{k} \\
& =-\sum_{n=1}^{\infty}\left(\sum_{k \mid n} \frac{1}{k}\right) e^{2 \pi i n z}=-\sum_{n=1}^{\infty} \sigma_{-1}(n) e^{2 \pi i n z}
\end{aligned}
$$


so that by (19)

$$
\log \eta(z)-\frac{\pi i z}{12}=-A(z, 0) .
$$

II. Let $\Gamma$ denote the (inhomogeneous) modular group-no confusion with the $\Gamma$ function should arise-that is, the group of fractional linear transformations $z \rightarrow(a z+b) /(c z+d)$, with $a, b, c, d$ integers and $a d-b c=1$. We' recall that $\Gamma$ maps $\mathcal{H}$ onto itself. Closely related to $\Gamma$ is the group $\pi$ of $2 \times 2$ integer matrices with determinant 1 . There is a homomorphism from $M$ onto $\Gamma$ which assigns to $\left(\begin{array}{ll}a & b \\ c & d\end{array}\right) \in \mathbb{M}$ the transformation $z \rightarrow(a z+b) /(c z+d)$. The kernel of the homomorphism is $\{E,-E\}$, where $E=\left(\begin{array}{ll}1 & 0 \\ 0 & 1\end{array}\right)$ is the identity matrix and $-\left(\begin{array}{ll}a & b \\ c & d\end{array}\right)=$ $\left(\begin{array}{ll}-a & -b \\ -c & -d\end{array}\right)$. In this section we are interested in determining the behavior of the function $G$ when $z \in \mathcal{H}$ is subjected to a transformation $V \in \Gamma$. The analysis simplifies considerably if we choose a particular one of the two possible matrices in $\mathbb{M}$ representing $V$. Thus, for given $V \in \Gamma$ we choose the unique $\left(\begin{array}{ll}a & b \\ c & d\end{array}\right) \in \mathbb{M}$ having $c>0$ or $c=0, d=1$ such that $V(z)=(a z+b) /(c z+d)$ and call it the standard representative of $V$, denoted $\underline{V}$. Observe that the set $\{\underline{V}\}, V$ ranging over $\Gamma$, is not a subgroup of $M$ and $V \rightarrow \underline{V}$ is not a homomorphism.

Theorem 2. Let

$$
V \in \Gamma, \quad \underline{V}=\left(\begin{array}{ll}
a & b \\
c & d
\end{array}\right), \quad c>0 \text { or } c=0, d=1,
$$

its standard representative. For $\left(r_{1}, r_{2}\right) \in R^{2}$, set

$$
\left(R_{1}, R_{2}\right)=\left(r_{1}, r_{2}\right) \underline{V}
$$

the ordinary matrix product.

(25) If $c=0, G\left(V(z), s, r_{1}, r_{2}\right)=G\left(z, s, R_{1}, R_{2}\right)$ for all $(z, s) \in \mathcal{H} \times C$.

(26) If $c>0$,

$\frac{G\left(V(z), s, r_{1}, r_{2}\right)}{(c z+d)^{s}}=G\left(z, s, R_{1}, R_{2}\right)$

where

$$
-2 i \sin \pi s\left\{\chi\left(R_{1}\right) \zeta\left(s,-R_{2}\right)+K\left(z, s, R_{1}, R_{2}, c, d\right)\right\}
$$

$$
K\left(z, s, R_{1}, R_{2}, c, d\right)=\sum_{m>R_{1}} \sum_{n>R_{2}+d\left(m-R_{1}\right) / c}\left(\left(m-R_{1}\right) z+n-R_{2}\right)^{-s}
$$

for all $(z, s) \in \mathcal{H} \times K_{2}$.

Proof. If $c=0, V(z)=z+b$ and for $\sigma>2$,

$$
\begin{aligned}
G\left(V(z), s, r_{1}, r_{2}\right) & =\sum_{m, n}^{\prime}\left(\left(m+r_{1}\right)(z+b)+n+r_{2}\right)^{-s} \\
& =\sum_{m, N}\left(\left(m+r_{1}\right) z+N+r_{1} b+r_{2}\right)^{-s}
\end{aligned}
$$


since as $(m, n)$ ranges over $Z^{2}$ omitting $\left(-r_{1},-r_{2}\right),(m, N), N=m b+n$, ranges over $Z^{2}$ omitting $\left(-r_{1},-r_{1} b-r_{2}\right)$. Now in this instance $\left(R_{1}, R_{2}\right)=$ $\left(r_{1}, r_{2}\right)\left(\begin{array}{ll}1 & b \\ 0 & 1\end{array}\right)=\left(r_{1}, r_{1} b+r_{2}\right)$ and we see that (25) halds, at least for $(z, s) \in \mathcal{H} \times$ $K_{2}$. As both sides of (25) have continuations to $(z, s) \in \mathcal{H} \times C$ the equality persists.

If $c>0$, we write, again for $\sigma>2$,

$$
\begin{aligned}
G\left(V(z), s, r_{1}, r_{2}\right) & =\sum_{m, n}\left(\left(m+r_{1}\right)\left(\frac{a z+b}{c z+d}\right)+n+r_{2}\right)^{-s} \\
& =\sum_{M, N}\left(\frac{\left(M+R_{1}\right) z+N+R_{2}}{c z+d}\right)^{-s}
\end{aligned}
$$

where $M=m a+n c, N=m b+n d$, i.e. $(M, N)=(m, n) \underline{V}$, which ranges over $Z^{2}$ omitting $\left(-R_{1},-R_{2}\right)$ as $(m, n)$ ranges over $Z^{2}$ omitting $\left(-r_{1},-r_{2}\right)$. Applying Lemma 1 (b), with $A=M+R_{1}, B=N+R_{2}, C=c>0, D^{\prime}=d$, we have that

$$
\left(\frac{\left(M+R_{1}\right) z+N+R_{2}}{c z+d}\right)^{-s}=e^{-2 \pi i s} \frac{\left(\left(M+R_{1}\right) z+N+R_{2}\right)^{-s}}{(c z+d)^{-s}}
$$

if $M+R_{1} \leq 0, d\left(M+R_{1}\right)>c\left(N+R_{2}\right)$ and $=\left(\left(M+R_{1}\right) z+N+R_{2}\right)^{-s} /(c z+d)^{-s}$, otherwise. Thus,

$$
\frac{G\left(V(z), s, r_{1}, r_{2}\right)}{(c z+d)^{s}}
$$

$$
=\sum_{1}\left(\left(M+R_{1}\right) z+N+R_{2}\right)^{-s}+e^{-2 \pi i s} \sum_{2}\left(\left(M+R_{1}\right) z+N+R_{2}\right)^{-s}
$$

where $\Sigma_{2}$ is the sum over those $(M, N)$ such that $M+R_{1} \leq 0, d\left(M+R_{1}\right)>$ $c\left(N+R_{2}\right)$ and $\Sigma_{1}$ the sum over the remaining $(M, N)$. Using an abbreviated notation the right. side of (28) may be written as $\Sigma_{1}+e^{-2 \pi i s} \Sigma_{2}=\Sigma_{1}+\Sigma_{2}+$ $\left(e^{-2 \pi i s}-1\right) \Sigma_{2}$ and $\Sigma_{1}+\Sigma_{2}=G\left(z, s, R_{1}, R_{2}\right)$. Since $c>0$,

$$
\begin{aligned}
\sum_{2} & =\sum_{M \leq-R_{1}} \sum_{N<-R_{2}+d\left(M+R_{1}\right) y / c}\left(\left(M+R_{1}\right) z+N+R_{2}\right)^{-s} \\
& =e^{\pi i s} \sum_{M \leq-R_{1}} \sum_{N<-R_{2}+d\left(M+R_{1}\right) / c}\left(\left(-M-R_{1}\right) z-N-R_{2}\right)^{-s} \\
& =e^{\pi i s} \sum_{m \geq R_{1}} \sum_{n>R_{2}+d\left(m-R_{1}\right) / c}\left(\left(m-R_{1}\right) z+n-R_{2}\right)^{-s}
\end{aligned}
$$


where in the last step we took $m=-M, n=-N$ as our summation variables. If $R_{1}$ is an integer we split off the terms corresponding to $m=R_{1}$ which contribute $\Sigma_{n>R_{2}}\left(n-R_{2}\right)^{-s}=\zeta\left(s,-R_{2}\right)$. (28) now is

$$
\begin{aligned}
& \frac{G\left(V(z), s, r_{1}, r_{2}\right)}{(c z+d)^{s}}=G\left(z, s, R_{1}, R_{2}\right)+\left(e^{-2 \pi i s}-1\right) e^{\pi i s} \\
& \left\{\chi\left(R_{1}\right) \zeta\left(s,-R_{2}\right)\right. \\
& \left.+\sum_{m>R_{1}} \sum_{n>R_{2}+d\left(m-R_{1}\right) / c}\left(\left(m-R_{1}\right) z+n-R_{2}\right)^{-s}\right\},
\end{aligned}
$$

which taking account of the definition of $K$ by $(27)$ and $\left(e^{-2 \pi i s}-1\right) e^{\pi i s}=$ $-2 i \sin \pi s$, proves (26).

The function $K$ is analytic on $\mathcal{H} \times \mathcal{K}$, it being just the sum of a subseries of the series defining $G$ and (26) shows that $K$ must have a continuation to $\mathcal{H} \times C$. Our goal is now to find, independently of (26), the continuation of $K$ and our method will be to imitate as well as possible one of the methods used for the $\zeta$ function. The basic tool is the identity $\Gamma(s) / \lambda^{s}=\int_{0}^{\infty} u^{s-1} e^{-\lambda u} d u$, valid for complex $\lambda$ having $\operatorname{Re} \lambda>0$ and $\sigma>0$. In fact, for $\lambda$ positive this is immediate from Euler's integral for $\Gamma(s)$ and both sides are analytic functions of $\lambda$ in the half plane $\operatorname{Re} \lambda>0$ so the equality persists. The restriction $\operatorname{Re} \lambda>0$ is needed to assure convergence of the integral. We can thus set $\lambda=\left(m-R_{1}\right) z+\left(n-R_{2}\right)$, for $z \in \mathcal{H}$, provided $\operatorname{Re}\left(\left(m-R_{1}\right) z+n-R_{2}\right)=\left(m-R_{1}\right) x+\left(n-R_{2}\right)$ is positive. In the sum for $K$, we have $n-R_{1}>0, n-R_{2}>d\left(m-R_{1}\right) / c$ so $\left(m-R_{1}\right) x+$ $\left(n-R_{2}\right)>\left(m-R_{1}\right)(x+d / c)$ which is positive provided $x+d / c>0$. Thus if $Q$ is the 'quadrant' $\{x>-d / c, y>0\}$ we have, for $(z, s) \in Q \times K_{2}$,

$$
\Gamma(s) K\left(z, s, R_{1}, R_{2}, c, d\right)
$$

$$
=\sum_{m>R_{1}} \sum_{n>R_{2}+d\left(m-R_{1}\right) / c} \int_{0}^{\infty} u^{s-1} \exp \left(-\left(m-R_{1}\right) z u-\left(n-R_{2}\right) u\right) d u
$$

We must now come to grips with the double sum and write it in a more mangeable form. Set, for $i=1,2$,

$$
g_{i}=\left[R_{i}\right], \quad \rho_{i}=\left\langle R_{i}\right\rangle,
$$

so that $R_{i}=g_{i}+\rho_{i}, g_{i} \in Z$ and $0 \leq \rho_{i}<1$. Then $m>R_{1}$ if and only if $m=g_{1}+$ $k, k \geq 1$. If $k=q c+j, 0 \leq j \leq c-1$, then $q \geq 0$ for $j>0$ and $q \geq 1$ for $j=0$. and $m=g_{1}+q c+j, m-R_{1}=q c+j-\rho_{1}$. Also $n>R_{2}+d\left(m-R_{1}\right) / c=g_{2}+$ $d q+\rho_{2}+d\left(j-\rho_{1}\right) / c$ if and only if $n=g_{2}+d q+\beta_{j}+b$, where $b$ is an integer $\geq 1$, and 


$$
\beta_{j}=\left[\rho_{2}+d\left(j-\rho_{1}\right) / c\right], \quad 0 \leq j \leq c-1
$$

Altogether then, $m>R_{1}, n>R_{2}+d\left(m-R_{1}\right) / c$ exactly for $m=g_{1}+q c+j, q \geq$ 0 for $1 \leq j \leq c-1, q \geq 1$ for $j=0, n=g_{2}+d q+\beta_{j}+b, b \geq 1$. For $x>-d / c$ we have seen that $\left(m-R_{1}\right) x+n-R_{2}$ is positive; hence for $x>-d / c$ and all admissible $j, q, b$,

$$
\left(q c+j-\rho_{1}\right) x+d q+\beta_{j}+b-\rho_{2}>0 .
$$

We can now rewrite (29), calling the left side just $\Gamma K$, splitting off by themselves the terms corresponding to $q=0$ for $j>0$, and also interchanging the order of summation and integration which will be justified momentarily, as

$$
\Gamma K=\sum_{j=0}^{c-1} \int_{0}^{\infty} u^{s-1} \sum_{q=1}^{\infty} \sum_{b=1}^{\infty} \exp \left(-\left(q c+j-\rho_{1}\right) z u-\left(d q+\beta_{j}+b-\rho_{2}\right) u\right) d u
$$

$$
+\sum_{j=0}^{c-1} \int_{0}^{\infty} u^{s-1} \sum_{b=1}^{\infty} \exp \left(-\left(j-\rho_{1}\right) z u-\left(\beta_{j}+b-\rho_{2}\right) u\right) d u
$$

Of course, if $c=1$ there are no $j>0$ and the sum $\Sigma_{j=1}^{c-1}$ is understood to be zero. In (33) if we replace each term by its absolute value this has the effect of replacing $s$ by $\sigma$ and $z$ by $x$. After a little simplification and summation of the geometric series one obtains

$$
\begin{aligned}
& \sum_{j=0}^{c-1} \int_{0}^{\infty} u^{\sigma-1} \frac{\exp \left(\left(\left(\rho_{1}-j\right) x+\rho_{2}-\beta_{j}\right) u\right)}{\left(e^{(c z+d) u}-1\right)\left(e^{u}-1\right)} d u \\
& \quad+\sum_{j=1}^{c-1} \int_{0}^{\infty} u^{\sigma-1} \frac{\exp \left(\left(\left(\rho_{1}-j\right) x+\rho_{2}-\beta_{j}\right) u\right)}{\left(e^{u}-1\right)} d u .
\end{aligned}
$$

Since $x>-d / c, \sigma>2$ is our standing assumption, the integrands are all positive and the integrals converge at $u=0$. At $u=\infty$ the integrands are comparable to $u^{\sigma-1} e^{-B u}$ with $B=\left(j-\rho_{1}\right) x+\beta_{j}-\rho_{2}+c x+d+1$, for $j \geq 0$ and $B=\left(j-\rho_{1}\right) x+$ $\beta_{j}-\rho_{2}+1$ for $j>0$. If $B>0$ the integrals converge, and this is indeed true as is seen by taking $q=1, b=1$ for $j \geq 0$ and $q=0, b=1$ for $j>0$, in (32). Thus in (33) the series and integrals are absolutely convergent and the change of order of sum and integral is justified. To simplify the notation, define

$$
Z=c z+d, \quad Y_{j}=\left(\rho_{1}-j\right) z+\rho_{2}-\beta_{j}, \quad 0 \leq j \leq c-1,
$$

and (33) becomes

$$
\Gamma K=\sum_{j=0}^{c-1} \int_{0}^{\infty} u^{s-1} \frac{\exp \left(Y_{j} u\right)}{\left(e^{Z u}-1\right)\left(e^{u}-1\right)} d u+\sum_{j=1}^{c-1} \int_{0}^{\infty} u^{s-1} \frac{\exp \left(Y_{j} u\right)}{\left(e^{u}-1\right)} d u
$$


These integrals are of the type known as Mellin transforms and the analytic continuation of $K$ is obtained as a special case of the following lemma. First, though, a few definitions. If $\phi(w)$ is a meromorphic function, not identically zero, in a domain containing the origin then ord ${ }_{w=0} \phi(w)=\nu, \nu$ an integer, means that $\phi$ has at $w=0$ a zero of multiplicity $\nu$ if $\nu>0$, a pole of multiplicity $-\nu$ if $\nu<0$, and neither zero nor pole if $\nu=0$. In any case, $w^{-\nu} \phi(w)$ is finite and nonzero at $w=0$. For our present purpose it is convenient to use the $s$ th power of $w$ arising from the value of $\operatorname{Arg} w$ between 0 and $2 \pi$. Thus we set $\left(w^{s}\right)=w^{s}$ if $0<\arg w<\pi$ and $\left(w^{s}\right)=e^{2 \pi i s} w^{s}$ if $-\pi \leq \arg w<0$. Let $E_{+}$, $E_{-}$denote the 'upper' and 'lower' 'edges', respectively, of the positive $u$ axis on which we define $\left(w^{s}\right)$ by continuity: if $w=u>0$ then on $E_{+},\left(w^{s}\right)=u^{s}$ and on $E_{-},\left(w^{s}\right)=$ $e^{2 \pi i s} u^{s}$. For $\delta>0$, let $\gamma=\gamma_{\delta}$ be the curve consisting of $E_{+}$from $+\infty$ to $\delta$, counterclockwise around $C_{\delta}=\{|w|=\delta\}$ and back along $E_{-}$from $\delta$ to $+\infty$.

Lem ma 3. Let $\phi(w)$ be a meromorpbic function not identically zero, in a domain containing the ray $\{u \geq 0, v=0\}$ and finite at all points of the positive axis $\{u>0, v=0\}$. Suppose ord ${ }_{w=0} \phi(w)=\nu$,

$$
w^{-\nu} \phi(w)=\sum_{j=0}^{\infty} c_{j} w^{j}, \quad c_{0} \neq 0,
$$

the series converging for $|w|<\delta_{0}\left(0<\delta_{0} \leq \infty\right)$.

If for some $\mu,-\nu<\mu \leq \infty, \phi$ satisfies $\phi(u)=O\left(u^{-9}\right)$ as $u \rightarrow \infty$, for every $\sigma$ in the interval $-\nu<\sigma<\mu$, then the transform

$$
\Phi(s)=\int_{0}^{\infty} u^{s-1} \phi(u) d u
$$

is an analytic function of $s$ in the strip $\{-\nu<\sigma<\mu\}-$ balf plane if $\mu=\infty$. $\Phi(s)$ bas a continuation to the balf plane $\{\sigma<\mu\}$-entire plane if $\mu=\infty$-given by the identity, valid for $-\nu<\sigma<\mu$,

$$
\Phi(s)=\frac{\Phi^{*}(s)}{\left(e^{2 \pi i s}-1\right)}
$$

where

$$
\Phi^{*}(s)=\int_{\gamma_{\delta}}\left(w^{s-1}\right) \phi(w) d w
$$

is analytic in $\{\sigma<\mu\}, \delta$ being any number in the interval $0<\delta<\delta_{0}$. Furthermore, if $s=-\nu-g$ is an integer $<\mu$ then

$$
\Phi^{*}(-\nu-g)=0, \quad \text { if } g<0, \quad \Phi^{*}(-\nu-g)=2 \pi i c_{g}, \quad \text { if } g \geq 0,
$$

$c_{g}$ being that obtained from (36).

Proof. Standard estimates show the existence and analyticity of $\Phi(s)$; the 
growth condition on $\phi(u)$ as $u \rightarrow \infty$ gives the convergence at $\infty$ of (37) and $\sigma>$ $-\nu$ assures convergence at 0 . In the same way it is easily seen that $\Phi^{*}(s)$, as defined by (39), is analytic in $\{\sigma<\mu\}$ and by the residue theorem is independent of $\delta$ in $0<\delta<\delta_{0}$. Breaking up $\gamma_{\delta}$ into two parts, the one along the axis and the other along $C_{\delta}$ gives

$$
\Phi^{*}(s)=\left(e^{2 \pi i s}-1\right) \int_{\delta}^{\infty} u^{s-1} \phi(u) d u+\int_{C_{\delta}}\left(w^{s-1}\right) \phi(w) d w .
$$

If $\delta$ is sufficiently small, $\left|w^{-\nu} \phi(w)\right| \leq 2\left|c_{0}\right|$ for $|w| \leq \delta$ and $\left|\int_{C_{\delta}}\left(w^{s-1}\right) \phi(w) d w\right|$ $\leq 4 \pi\left|c_{0}\right| e^{2 \pi|t|} \delta^{\sigma+\nu}$, hence if $\sigma>-\nu$, as $\delta \rightarrow 0$ in (41), $\int_{C_{\delta}} \rightarrow 0$, and

$$
\Phi^{*}(s)=\left(e^{2 \pi i s}-1\right) \int_{0}^{\infty} u^{s-1} \phi(u) d u=\left(e^{2 \pi i s}-1\right) \Phi(s),
$$

which gives the continuation of $\Phi(s)$ as stated by (38). Note that since $\Phi^{*}(s)$ is analytic in $\{\sigma<\mu\}$, the extended $\Phi(s)$ picks up at most simple poles at $s=$ integer $\leq-\nu$. Finally, for $s=-\nu-g$ an integer $<\mu$, by $(41), \Phi^{*}(-\nu-g)=$ $\int c_{\delta} w^{-\nu-8-1} \phi(w) d w$, for when $s$ is an integer $\left(w^{s}\right)=w^{s}$, and the latter integral is just $2 \pi i \operatorname{Re} s_{w=0} w^{-\nu-8-1} \phi(w)$, which, by (36), gives (40). This proves the lemma.

Keeping the notation established before Lemma 3 we summarize our results concerning $G$ and $K$, all pertaining to the case $c>0$.

Theorem 3. Let $Y_{j}, Z$ be defined by (34),

$$
\phi\left(Y_{j}, Z, w\right)=\exp \left(Y_{j} w\right) /\left(e^{Z w}-1\right)\left(e^{w}-1\right), \quad 0 \leq j \leq c-1,
$$

and

Let

$$
\psi\left(Y_{j}, w\right)=\exp \left(Y_{j} w\right) /\left(e^{w}-1\right), \quad 1 \leq j \leq c-1
$$

$$
\begin{aligned}
\Phi\left(Y_{j}, Z, s\right) & =\int_{0}^{\infty} u^{s-1} \phi\left(Y_{j}, Z, u\right) d u, \\
\Psi\left(Y_{j}, s\right) & =\int_{0}^{\infty} u^{s-1} \psi\left(Y_{j}, u\right) d u, \\
\Phi^{*}\left(Y_{j}, Z, s\right) & =\int_{\gamma_{\delta}}\left(w^{s-1}\right) \phi\left(Y_{j}, Z, w\right) d w, \\
\Psi^{*}\left(Y_{j}, s\right) & =\int_{\gamma_{\delta}}\left(w^{s-1}\right) \psi\left(Y_{j}, w\right) d w,
\end{aligned}
$$

where $0<\delta<\delta_{0}=\min (2 \pi, 2 \pi /|Z|)$.

Then $\Phi^{*}, \Psi^{*}$ are entire functions of $s, \Phi, \Psi$ are analytic in the balf plane $K_{2}, K_{1}$ respectively, and bave continuations over the whole $s$ plane given by

$$
\Phi\left(Y_{j}, Z, s\right)=\frac{\Phi^{*}\left(Y_{j}, Z, s\right)}{\left(e^{2 \pi i s}-1\right)}, \quad \Psi\left(Y_{i}, s\right)=\frac{\Psi^{*}\left(Y_{j}, s\right)}{\left(e^{2 \pi i s}-1\right)},
$$

valid in $\mathcal{K}_{2}, \mathcal{K}_{1}$ respectively. 
If $Q$ is the quadrant $\{x>-d / c, y>0\}$ then for $(z, s) \in Q \times K_{2}$,

$$
\Gamma(s) K\left(z, s, R_{1}, R_{2}, c, d\right)=\sum_{j=0}^{c-1} \Phi\left(Y_{j}, Z, s\right)+\sum_{j=1}^{c-1} \Psi\left(Y_{j}, s\right)
$$

and, for $(z, s) \in Q \times C$,

$$
\left(e^{2 \pi i s}-1\right) \Gamma(s) K\left(z, s, R_{1}, R_{2}, c, d\right)=\sum_{j=0}^{c-1} \Phi *\left(Y_{i}, Z, s\right)+\sum_{j=1}^{c-1} \Psi *\left(Y_{i}, s\right) .
$$

Finally, for $(z, s) \in Q \times C$,

$$
\begin{aligned}
\frac{G\left(V(z), s, r_{1}, r_{2}\right)}{(c z+d)^{s}}= & G\left(z, s, R_{1}, R_{2}\right)-\chi\left(R_{1}\right) 2 i(\sin \pi s) \zeta\left(s,-R_{2}\right) \\
& -\frac{e^{-\pi i s}}{\Gamma(s)}\left\{\sum_{j=0}^{c-1} \Phi^{*}\left(Y_{j}, Z, s\right)+\sum_{j=1}^{c-1} \Psi^{*}\left(Y_{j}, s\right)\right\} .
\end{aligned}
$$

Proof. The $\phi, \psi$ are meromorphic in the whole $w$-plane; each $\psi$ has a simple pole at the origin, ord ${ }_{w=0} \psi=-1$, and is finite in $0<|w|<2 \pi$, while each $\phi$ has a double pole at the origin, ord ${ }_{w=0} \phi=-2$, and is finite in $0<|w|<$ $\min (2 \pi, 2 \pi /|Z|)$ and now the statements concerning $\Phi, \Phi^{*}$ follow immediately from Lemma 3. (42), (43) are just reformulations of (35) while (44) arises upon substitution of $K$, as given by (43), into (26).

The formula (44) is somewhat complicated and furthermore has the drawback that it holds only for $z$ restricted to the quadrant $Q$ since otherwise the integrals defining $\Phi^{*}$ do not converge.

When $s$ is an integer, though, we can simplify (44) and also obtain a formula valid for all $z \in \mathcal{H}$. First, recall that at the end of $\$ I$ we showed that for $s=$ integer $\nu \leq 0, G\left(z, \nu, r_{1}, r_{2}\right)$ is the constant function in $z, \chi\left(r_{1}\right)\left(\zeta\left(\nu, r_{2}\right)+(-1)^{\nu} \zeta\left(\nu,-r_{2}\right)\right)$. Now by (44), since $1 / \Gamma(\nu)=0$, sin $\pi \nu=0$ and the remaining functions of $s$ are analytic at $s=\nu$, we obtain

$$
G\left(V(z), \nu, r_{1}, r_{2}\right) /(c z+d)^{\nu}=G\left(z, \nu, R_{1}, R_{2}\right) .
$$

Consider the case $V(z)=-1 / z, \underline{V}=\left(\begin{array}{cc}0 & -1 \\ 1 & 0\end{array}\right),\left(r_{1}, r_{2}\right)=(0, r), r$ any real number, so that $\left(R_{1}, R_{2}\right)=(0, r) \underline{V}=(r, 0)$ and the above formula becomes

$$
G(-1 / z, \nu, 0, r) / z^{\nu}=G(z, \nu, r, 0) .
$$

Now replace each $G$ by its constant value as given above and we have

$$
\zeta(\nu, r)+(-1)^{\nu} \zeta(\nu,-r) / z^{\nu}=\chi^{(r)}\left(\zeta(\nu)+(-1)^{\nu} \zeta(\nu)\right) .
$$

We have already previously remarked that $\zeta(\nu)+(-1)^{\nu} \zeta(\nu)=0$ if $\nu<0$ and $=-1$ if $\nu=0$. Thus 


$$
\begin{aligned}
& \text { for any real } r \text { and integer } \nu<0, \\
& \zeta(\nu, r)+(-1)^{\nu} \zeta(\nu,-r)=0 \text { while } \\
& \zeta(0, r)+\zeta(0,-r)=-\chi(r) .
\end{aligned}
$$

By Lemma 3 we can evaluate the $\Phi^{*}$ and $\Psi^{*}$ at any integer value of $s$. In fact, by that lemma, since

$$
\operatorname{ord}_{w=0} \frac{\exp \left(Y_{j} w\right)}{\left(e^{Z w}-1\right)\left(e^{w}-1\right)}=-2 \text { and } \operatorname{ord}_{w=0} \frac{\exp \left(Y_{j} w\right)}{\left(e^{w}-1\right)}=-1 \text {, }
$$

$\Phi^{*}\left(Y_{j}, Z, \nu\right)=0$ for $\nu$ an integer $\geq 3, \Psi^{*}\left(Y_{j}, \nu\right)=0$ for $\nu$ an integer $\geq 2$, while for $g$ an integer $\geq 0, \Phi^{*}\left(Y_{j}, Z, 2-g\right)=2 \pi i$ times the coefficient of $w^{8}$ in the expansion of $w^{2} \exp \left(Y_{j} w\right) /\left(e^{Z w}-1\right)\left(e^{w}-1\right)$ about $w=0$ and $\Psi *\left(Y_{j}, 1-g\right)=2 \pi i$ times the coefficient of $w^{g}$ in the expansion of $w \exp \left(Y_{j} w\right) /\left(e^{w}-1\right)$ about $w=0$. Now $w /\left(e^{w}-1\right)=\Sigma_{n}^{\infty}=0 b_{n} w^{n} / n$ !, for $|w|<2 \pi$, the $b_{n}$ being the Bernoulli numbers, $b_{0}=1, b_{1}=-1 / 2, b_{2}=1 / 6$ and $b_{2 k+1}=0$ for $k \geq 1$. Hence

$$
\begin{aligned}
\frac{w^{2} \exp \left(Y_{j} w\right)}{\left(e^{Z w}-1\right)\left(e^{w}-1\right)} & =\frac{1}{Z} \cdot \exp \left(Y_{j} w\right) \cdot \frac{w}{e^{w}-1} \cdot \frac{Z w}{e^{Z w}-1} \\
& =\frac{1}{Z} \sum_{k=0}^{\infty} \frac{Y_{j}^{k}}{k !} w^{k} \sum_{n=0}^{\infty} \frac{b_{n}}{n !} w^{n} \sum_{p=0}^{\infty} \frac{b_{p}}{p !} Z^{p} w^{p}, \\
\exp (Y, w) \cdot \frac{w}{e^{w}-1} & =\sum_{k=0}^{\infty} \frac{Y_{j}^{k}}{k !} w^{k} \sum_{n=0}^{\infty} \frac{b_{n}}{n !} w^{n},
\end{aligned}
$$

and so

$$
\Phi^{*}\left(Y_{i}, Z, 2-g\right)=\frac{2 \pi i}{Z} L_{g}\left(Y_{j}, Z\right), \quad \Psi^{*}\left(Y_{j}, 1-g\right)=2 \pi i J_{g}\left(Y_{j}\right)
$$

where

$$
\begin{gathered}
L_{8}\left(Y_{j}, Z\right)=\sum_{k+n+p=8} \frac{Y_{j}^{k}}{k !} \frac{b_{n}}{n !} \frac{b_{p}}{p !} Z^{p} \\
J_{g}\left(Y_{j}\right)=\sum_{k+n=8} \frac{Y_{j}^{k}}{k !} \frac{b_{n}}{n !} .
\end{gathered}
$$

Each $Y_{j}, Z$ was a linear polynomial in $z$ so that $L_{g^{\prime}} J_{g}$ are also polynomials in $z$. It follows now from (44) and the above that

$$
\left.G\left(V(z), \nu, r_{1}, r_{2}\right) /(c z+d)^{\nu}=G(z, \nu) R_{1}, R_{2}\right), \text { for } s=\nu \geq 3,
$$

which of course is classical and can be obtained directly from the definition of $G$. Also, since $Z=c z+d, L_{0}\left(Y_{j}, Z\right)=1, J_{0}\left(Y_{j}\right)=1, L_{1}\left(Y_{j}, Z\right)=Y_{j}-1 / 2-(1 / 2) Z$, $\sin 2 \pi \zeta\left(2,-R_{2}\right)=0,\left.\sin (\pi s) \cdot \zeta\left(s,-R_{2}\right)\right|_{s=1}=-\pi$ (recall that $\zeta\left(s,-R_{2}\right)=$ $1 /(s-1)+\cdots$ near $s=1)$ we have 


$$
\begin{aligned}
\frac{G\left(V(z), 2, r_{1}, r_{2}\right)}{(c z+d)^{2}}= & G\left(z, 2, R_{1}, R_{2}\right)-\frac{2 \pi \mathrm{ic}}{(c z+d)}, \\
\frac{G\left(V(z), 1, r_{1}, r_{2}\right)}{(c z+d)}= & G\left(z, 1, R_{2}, R_{2}\right)+2 \pi i \chi\left(R_{1}\right)+\frac{2 \pi i}{(c z+d)} \sum_{j=0}^{c-1} Y_{j} \\
& -\frac{\pi i c}{c z+d}+\pi i(c-2) .
\end{aligned}
$$

The above holds for all $z \in \mathcal{H}$; for by (44) they are valid for $z \in Q$ but all the functions involved are analytic for $z \in \mathcal{H}$, so the equality persists. Concerning (49) we note that it is remarkable in that the extra term added on at the right is independent of $r_{1}, r_{2}$. Also, (49) holds even if $c=0$, as may be seen by comparison with (25). Furthermore, we need not use the standard matrix representative

$$
\underline{V}=\left(\begin{array}{ll}
a & b \\
c & d
\end{array}\right)
$$

of $V$, for using instead $-\underline{V}$, both $(c z+d)^{2}, c /(c z+d)$ are unchanged, while $G\left(z, 2, R_{1}, R_{2}\right)$ is replaced by $G\left(z, 2,-R_{1},-R_{2}\right)$. But these are equal, as may be seen from (18) with $s=2$.

Along with $G$ it is interesting to consider the function $H\left(z, s, r_{1}, r_{2}\right)=$ $A\left(z, s, r_{1}, r_{2}\right)+e^{\pi i s} A\left(z, s,-r_{1},-r_{2}\right) ; A$ being defined by (17). By (18) we have $G\left(z, s, r_{1}, r_{2}\right)=\chi\left(r_{1}\right)\left(\zeta\left(s, r_{2}\right)+e^{\pi i s} \zeta\left(s,-r_{2}\right)\right)+\Delta(s) H\left(z, s, r_{1}, r_{2}\right)$. Now replace both occurrences of $G$ in (44) by the above expression involving $H$ and then solve for $H\left(V(z), s, r_{1}, r_{2}\right)$. After a little calculation, taking into account

$$
\Delta(s)=\frac{(-2 \pi i)^{s}}{\Gamma(s)}=\frac{e^{-\pi i s}(2 \pi i)^{s}}{\Gamma(s)},
$$

the result is

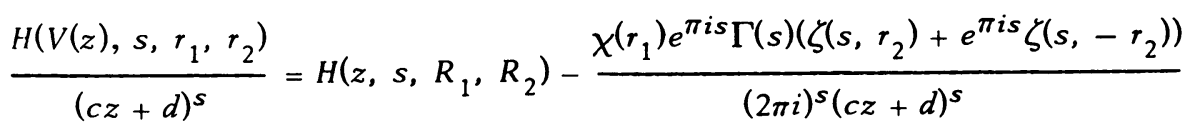

$$
\begin{aligned}
& +\frac{\chi\left(R_{1}\right) \Gamma(s)\left(\zeta\left(s,-R_{2}\right)+e^{\pi i s} \zeta\left(s, R_{2}\right)\right)}{(2 \pi i)^{s}} \\
& -\frac{1}{(2 \pi i)^{s}}\left\{\sum_{j=0}^{c-1} \Phi^{*}\left(Y_{j}, Z, s\right)+\sum_{j=1}^{c-1} \Psi^{*}\left(Y_{j}, s\right)\right\} \text {. }
\end{aligned}
$$

This holds for all $(z, s) \in Q \times C$. In case $\left(r_{1}, r_{2}\right) \in Z^{2}, H\left(z, s, r_{1}, r_{2}\right)=$ $\left(1+e^{\pi i s}\right) A(z, s)$, where $A(z, s)=A(z, s, 0,0)$, see (19) above, and we obtain the transformation formula for $A(z, s)$ : 


$$
\begin{aligned}
\frac{A(V(z), s)}{(c z+d)^{s}} & =A(z, s)+\frac{\Gamma(s) \zeta(s)}{(2 \pi i)^{s}}\left(1-\frac{e^{\pi i s}}{(c z+d)^{s}}\right) \\
& -\frac{1}{(2 \pi i)^{s}\left(1+e^{\pi i s}\right)}\left\{\sum_{j=0}^{c-1} \Phi *\left(Y_{j}, Z, s\right)+\sum_{j=1}^{c-1} \Psi *\left(Y_{j}, s\right)\right\} .
\end{aligned}
$$

In this and other formulas involving $A(z, s)$ it is understood that the $Y_{j}$ are computed using $\left(r_{1}, r_{2}\right)=\left(R_{1}, R_{2}\right)=(0,0)$.

Again these formulas can be simplified in case $s$ is an integer. If $s=\nu$, an integer, and $r$ any real number, let $\delta(\nu, r)=\Gamma(s)\left(\zeta(s, r)+e^{\pi i s} \zeta(s,-r)\right)$ evaluated at $s=\nu$. For $\nu>0$ both $\Gamma(s)$ and $\zeta(s, r)+e^{\pi i s} \zeta(s,-r)$ are finite at $s=\nu$ so $\delta(\nu, r)$ is well defined. For $\nu<0, \Gamma(s)$ has a simple pole at $s=\nu$ and $\zeta(s, r)+$ $e^{\pi i s} \zeta(s,-r)$ by $(45)$ has a zero at $s=\nu$ so again $\delta(\nu, r)$ is a finite number.

Similarly when $\nu=0$ as long as $r$ is not an integer, while $\delta(0, r)=\infty$ if $r \in Z$.

We remark that we cannot actually calculate $\delta$ for that involves knowing the values of $\partial \zeta(\nu, r) / \partial s$, which in general we (meaning the author) do not know. In any case, for $s=\nu \neq 0$ we have from (51) that

$$
\begin{aligned}
\frac{H\left(V(z), \nu, r_{1}, r_{2}\right)}{(c z+d)^{\nu}}= & H\left(z, \nu, R_{1}, R_{2}\right)-\frac{\chi^{\left(r_{1}\right)(-1)^{\nu} \delta\left(\nu, r_{2}\right)}}{(2 \pi i)^{\nu}(c z+d)^{\nu}}+\frac{\chi^{\left(R_{1}\right) \delta\left(\nu,-R_{2}\right)}}{(2 \pi i)^{\nu}} \\
& -\frac{1}{(2 \pi i)^{\nu}}\left\{\sum_{j=0}^{c-1} \Phi^{*}\left(Y_{j}, Z, \nu\right)+\sum_{j=1}^{c-1} \Psi *\left(Y_{j}, \nu\right)\right\},
\end{aligned}
$$

where $\Phi^{*}, \Psi^{*}$ are given by (46); (47) and are zero otherwise. By the remarks following (50), the above holds for all $z \in \mathcal{H}$. If $\nu=0$, (53) clearly still follows from

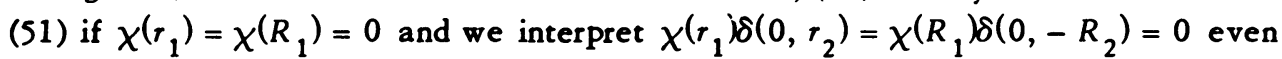
in case $r_{2} \in Z$ or $R_{2} \in Z$. The only case remaining then is if $\nu=0, r_{1} \in Z, r_{2}$ $\epsilon Z$-in which case necessarily then $R_{1}, R_{2} \in Z$-which means we are actually dealing with $A(z, 0)$. Thus let us now consider (52) for $s=\nu$; here the situation is somewhat more complicated. In fact we see that the individual terms in (52) to the right of $A(z, s)$ can have poles at $s=\nu$. Of course the total residue must be zero since $A(z, \nu), A(V(z), \nu) /(c z+d)^{\nu}$ are finite, but we have no way of computing independently the constant contributed by the remaining terms. If $\nu$ is even the only possible singularity can come from $\Gamma(s) \zeta(s)$ which is manageable. If $\nu=2 k \geq 4$, since $\Phi^{*}, \Psi^{*}$ vanish we have

$$
\frac{A(V(z), 2 k)}{(c z+d)^{2 k}}=A(z, 2 k)+\frac{(-1)^{k}(2 k-1) ! \zeta(2 k)}{(2 \pi)^{2 k}}\left(1-\frac{1}{(c z+d)^{2 k}}\right) .
$$

Again, this and the following formulas hold for all $z \in \mathcal{H}$, as remarked above. For $s=2$, since $\sum_{j=0}^{c=1} \Phi *\left(Y_{j}, Z, 2\right)=2 \pi i c / Z, \zeta(2)=\pi^{2} / 6$, 


$$
\frac{A(V(z), 2)}{(c z+d)^{2}}=A(z, 2)+\frac{1}{24}\left(\frac{1}{(c z+d)^{2}}-1\right)+\frac{i c}{4 \pi(c z+d)} .
$$

Expanding about $s=0$ one has

$$
\Gamma(s) \zeta(s)\left(1-\frac{e^{\pi i s}}{(c z+d)^{s}}\right)=\left(\frac{1}{s}+\cdots\right)\left(-\frac{1}{2}+\cdots\right)((\log (c z+d)-\pi i) s+\cdots)
$$

which at $s=0$ has the value $(\pi i-\log (c z+d)) / 2$. Thus

$$
\begin{aligned}
A(V(z), 0)= & A(z, 0)+\frac{\pi i}{2}-\frac{\log (c z+d)}{2} \\
& -\frac{1}{2}\left\{\sum_{j=0}^{c-1} \Phi *\left(Y_{j}, Z, 0\right)+\sum_{j=1}^{c-1} \Psi *\left(Y_{j}, 0\right)\right\} .
\end{aligned}
$$

By (46), (47), $\Phi^{*}\left(Y_{j}, Z, 0\right)=2 \pi i L_{2}\left(Y_{j}, Z\right) / Z$ and $L_{2}\left(Y_{j}, Z\right)=Y_{j}^{2} / 2-Y_{j} / 2-$ $Y_{j} Z / 2+1 / 12+Z / 4+Z^{2} / 12, \Psi *\left(Y_{j}, 0\right)=2 \pi i J_{1}\left(Y_{j}\right)$ and $J_{1}\left(Y_{j}\right)=Y_{j}-1 / 2$. Recalling the definitions of $Y_{j},(34)$ and $\beta_{j},(31)$, we see, since we have $\left(r_{1}, r_{2}\right)=$ $(0,0)$, that $\beta_{j}=[d j / c], Y_{j}=-j z-[d j / c], 0 \leq j \leq c-1$, hence $Y_{0}=0$. Putting the above into (56) and taking account of $\log \eta(z)=\pi i z / 12-A(z, 0)$, as shown in (22), we have, for $V(z)=(a z+b) /(c z+d), c>0$,

$$
\begin{aligned}
\log \eta\left(\frac{a z+b}{c z+d}\right)= & \log \eta(z)+\frac{\pi i}{12}\left(\frac{a z+b}{c z+d}-z\right)+\frac{\log (c z+d)}{2} \\
& +\frac{\pi i}{2(c z+d)} \sum_{j=1}^{c-1}\left(Y_{j}^{2}-Y_{j}\right)+\frac{\pi i c}{12(c z+d)} \\
& +\frac{\pi i}{2} \sum_{j=1}^{c-1} Y_{j}-\frac{\pi i c}{4}+\frac{\pi i c(c z+d)}{12}
\end{aligned}
$$

This does not appear in as elegent a form as obtained by Dedekind, but by actually carrying out the indicated summations one can recast (57) into the standard form involving the Dedekind sum. For the case $V(z)=-1 / z, c=1$, all the sums are absent and we have $\log \eta(-1 / z)=\log \eta(z)+(\log z) / 2-\pi i / 4$.

Finally, in case $s$ is a negative even integer, $s=-2 k, k \geq 1$, then using the functional equation of the $\zeta$ function one has $\Gamma(s) \zeta(s)_{s=-2 k}=(-1)^{k} \zeta(2 k+1) / 2(2 \pi)^{2 k}$ so that (52) yields

$$
\frac{A(V(z),-2 k)}{(c z+d)^{-2 k}}=A(z,-2 k)+\frac{\zeta(2 k+1)}{2}\left(1-(c z+d)^{2 k}\right)
$$

$$
+(-1)^{k+1}(2 \pi)^{2 k+1} \cdot \frac{i}{2}\left\{\frac{1}{Z} \sum_{j=0}^{c-1} L_{2 k+2}\left(Y_{j}, Z\right)+\sum_{j=1}^{c-1} J_{2 k+1}\left(Y_{j}\right)\right\}
$$


In the conclusion of this section we point out that some amusing and interesting calculations can be done with these formulas by putting in particular values of $z$. We shall leave this to the pleasure of the reader, pointing out one sample. In (55) take $z=i, V(z)=-1 / z$, so that $V(i)=i$, and solve for $A(i, 2)$ to obtain

$$
\sum_{n=1}^{\infty} \sigma_{1}(n) e^{-2 \pi n}=\frac{1}{24}-\frac{1}{8 \pi}
$$

Again taking $z=i, V(i)=(a i+b) /(c i+d), c>0$, gives

$$
\sum_{n=1}^{\infty} \sigma_{1}(n) e^{2 \pi i n((a i+b) /(c i+d))}=\frac{1}{24}-\frac{c^{2}+d^{2}}{8 \pi} .
$$

Since both sides are invariant under change of the signs of all of $a, b, c, d$, the above formula is seen to hold for any $\left(\begin{array}{ll}a & b \\ c & d\end{array}\right) \in \mathbb{N}$. Splitting the exponential into real and imaginary parts, one has

$$
\begin{aligned}
& \sum_{n=1}^{\infty} \sigma_{1}(n) \exp \left(-2 \pi n /\left(c^{2}+d^{2}\right)\right) \cos \frac{2 \pi n(a c+b d)}{c^{2}+d^{2}}=\frac{1}{24}-\frac{c^{2}+d^{2}}{8 \pi}, \\
& \sum_{n=1}^{\infty} \sigma_{1}(n) \exp \left(-2 \pi n /\left(c^{2}+d^{2}\right)\right) \sin \frac{2 \pi n(a c+b d)}{c^{2}+d^{2}}=0 .
\end{aligned}
$$

III. In this brief concluding section we exhibit the connection between the function $G\left(z, s, r_{1}, r_{2}\right)$ and the classical Eisenstein series, thus justifying the title of the paper. The latter are series of the form $\Sigma 1 /\left(m_{1} z+n_{1}\right)^{k}, k$ an integer, $\left(m_{1}, n_{1}\right)$ ranging over $Z^{2}$ subject to a restriction of the type $m_{1} \equiv a_{1}, n_{1} \equiv$ $a_{2} \bmod N, N$ a positive integer, $a_{1}, a_{2}$ arbitrary integers. These series converge only for $k \geq 3$, and if one sets $m_{1}=a_{1}+m N, n_{1}=a_{2}+n N$ one sees that the series can be written as

$$
\frac{1}{N^{k}} \sum_{m, n}^{\prime} \frac{1}{\left(\left(m+a_{1} / N\right) z+n+a_{2} / N\right)^{k}}=\frac{1}{N^{k}} G\left(z, k, \frac{a_{1}}{N}, \frac{a_{2}}{N}\right) .
$$

Thus for $s=k, r_{1}, r_{2}$ rational numbers, our $G$ function coincides essentially with the Eisenstein series. For $k=2$, the Eisenstein series do not converge and Hecke in [1] introduced the idea of considering instead

$$
\lim _{s \rightarrow 0} \sum^{\prime} \frac{1}{\left(m_{1} z+n_{1}\right)^{2}\left|m_{1} z+n_{1}\right|^{s}},
$$

$\left(m_{1}, n_{1}\right)$ subject to the same restriction as above. From our point of view one should consider instead the function $G\left(z, 2, a_{1} / N, a_{2} / N\right)$. The relation between the two is as follows. Hecke denotes the function defined by the above limit as $G_{2}\left(z ; a_{1}, a_{2}, N\right)$ and gives for it a Fourier expansion which, as easily seen, can be rewritten in our notation as 


$$
\begin{aligned}
G_{2}\left(z ; a_{1}, a_{2}, N\right)= & \frac{-2 \pi i}{N^{2}(z-\bar{z})}+\frac{1}{N^{2}} \times\left(\frac{a_{1}}{N}\right)\left(\zeta\left(2, \frac{a_{2}}{N}\right)+\zeta\left(2,-\frac{a_{2}}{N}\right)\right) \\
& -\frac{4 \pi^{2}}{N^{2}} \sum_{m>-a_{1} / N} \sum_{k=1}^{\infty} k \exp \left(2 \pi i k a_{2} / N\right) \exp \left(2 \pi i k\left(m+a_{1} / N\right) z\right) \\
& -\frac{4 \pi^{2}}{N^{2}} \sum_{m>a_{1} / N} \sum_{k=1}^{\infty} k \exp \left(-2 \pi i k a_{2} / N\right) \exp \left(2 \pi i k\left(m-a_{1} / N\right) z\right)
\end{aligned}
$$

which upon comparison with (17), (18) for $s=2$ shows that

$$
G_{2}\left(z ; a_{1}, a_{2}, N\right)=-2 \pi i / N^{2}(z-\bar{z})+N^{-2} G\left(z, 2, a_{1} / N, a_{2} / N\right) .
$$

From this one deduces easily how $G_{2}\left(z ; a_{1}, a_{2}, N\right)$ transforms under the modular group, for (49) shows how $G$ transforms, and remarkably the nonanalytic function $2 \pi i /(z-\bar{z})$ transforms the same way. In fact if $\left(\begin{array}{ll}a & b \\ c & d\end{array}\right)$ is any real matrix with determinant one an easy calculation shows that, if $f(z)=2 \pi i / z-\bar{z}$,

$$
\frac{f((a z+b) /(c z+d))}{(c z+d)^{2}}=f(z)-\frac{2 \pi i c}{c z+d} .
$$

Thus the extra added terms from $G$ and $-f$ cancel and we have Hecke's result that

$$
\frac{G_{2}\left((a z+b) /(c z+d) ; a_{1}, a_{2}, N\right)}{(c z+d)^{2}}=G_{2}\left(z ; a a_{1}+c a_{2}, b a_{1}+d a_{2}, N\right),
$$

for any $\left(\begin{array}{ll}a & b \\ c & d\end{array}\right) \in M$.

\section{BIBLIOGRAPHY}

1. E. Hecke, Theorie der Eisensteinschen Reihe hoherer Stufe und ihre Anwendung auf Funktionentheorie und Arithmetik, Abh. Math. Sem. Univ. Hamburg S (1927), 199-224.

2. J. Lewittes, Analytic continuation of the series $\Sigma(m+n z)^{-s}$, Trans. Amer. Math. Soc. 159 (1971), 594-598.

3. E. T. Whittaker and G. N. Watson, A course of modern analysis, Cambridge Univ. Press, New York, 1969 (reprint).

DEPARTMENT OF MATHEMATICS, HERBERT H. LEHMAN COLLEGE (CUNY), BRONX, NEW YORK 10468

DEPARTMENT OF MATHEMATICS, GRADUATE CENTER (CUNY), NEW YORK, NEW YORK 10036 (Current address) 